\title{
Submicroliter Electrochemistry and Spectroelectrochemistry Using Standard Electrodes and a Polymer Electrolyte Salt Bridge
}

\author{
Paul A. Flowers* and David A. Blake \\ Department of Chemistry and Physics, University of North Carolina at Pembroke, Pembroke, North Carolina 28372-1510, United \\ States
}

\begin{abstract}
The development of spectroscopic and electrochemical devices that can accommodate very small samples is of considerable importance to many areas of science and technology. We report here on the design and characteristics of a simple apparatus for the electrochemical and spectroelectrochemical analysis of submicroliter aqueous samples. The device is easily assembled from common laboratory materials and equipment, utilizing a bifurcated fiber-optic probe, standard disk electrodes of millimeter dimensions, and a polymer electrolyte film salt bridge to enable the analysis of nanoliter-scale sample volumes in a thin-layer configuration. Excellent performance has been demonstrated via measurements on aqueous ferricyanide solutions using sample volumes as low as $20 \mathrm{~nL}$.
\end{abstract}

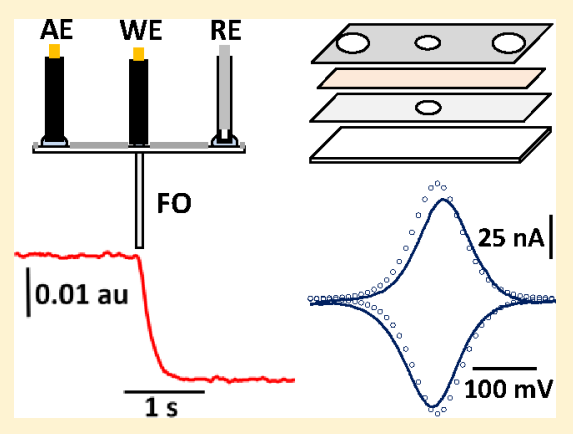

$\mathrm{D}$ eveloping new tools for spectroscopic and electrochemical analyses of increasingly small samples is a consistent research focus in analytical chemistry, given the benefit of such tools in applications where the sample is intrinsically small, scarce, hazardous, or expensive. Sampling accessories and dedicated instruments permitting spectral measurements in the ultraviolet through infrared regions are presently available from several vendors that can accommodate sample volumes on the order of $\sim 1 \mu \mathrm{L}$. Comparably fewer electrochemical cells suitable for small samples, on the order of a few hundred microliters, are commercially available, but there is a considerable research effort directed toward the development of electrochemical cells and sensors capable of analyzing very small volumes. ${ }^{1,2}$ In particular, electrochemical devices that can accommodate submicroliter sample volumes have been constructed using various microfabrication techniques, including traditional ultramicroelectrode preparation strategies, ${ }^{3,4}$ photo- and electron-beam lithography, ${ }^{5,6}$ and sequential screen printing/laser micromachining.

Additional challenges are encountered in the design of devices capable of simultaneous electrochemical and spectral measurements (spectroelectrochemistry or SEC), ${ }^{8}$ and there are consequently fewer reports describing SEC apparatus for microscale measurements. Small analysis volumes in SEC are most often achieved by isolating liquid samples between two closely spaced, parallel planar surfaces, at least one of which functions as a working electrode. Various optical sampling modes are possible depending upon the identities of these planar substrates: solid electrodes, optically transparent electrodes, windows, internal reflection crystals, etc. A relatively large separation of these planar surfaces yields a relatively low ratio of electrode area-to-solution volume $(\mathrm{A} / \mathrm{V})$, resulting in semiinfinite linear diffusion or "bulk" electrochemical behavior. Relatively small separations result in larger $\mathrm{A} / \mathrm{V}$ ratios and restricted diffusion conditions. In cases where the separation is lesser than the diffusion distance of the analyte on the experimental time scale, the electrochemical behavior is characterized by rapid equilibration of the redox couple concentrations to changes in the applied potential and is classified as "thin-layer". Cells of both bulk and thin-layer configurations have been designed for batch analysis of minimum sample volumes ranging from hundreds ${ }^{10-12}$ to tens of microliters. ${ }^{13-20}$

Recent work in our laboratory has focused on the development of SEC apparatus that use polymer electrolyte salt bridges to provide ionic contact between separate counter electrode solutions and very small volume samples. This strategy is similar in concept to that first employed by Mark et al. over 30 years ago in a thin-layer electrochemical cell capable of accommodating sample volumes as small as $0.25 \mu \mathrm{L},{ }^{21}$ but to the best of our knowledge, it has not been subsequently exploited for SEC cell designs. We previously reported on the design and performance of an SEC device requiring only simple machining for its construction and featuring an easily filled sample microwell with a platinum coil working electrode. The sample well and working electrode dimensions result in a moderate $\mathrm{A} / \mathrm{V}$ that yields bulk electrochemical behavior and enables SEC analysis of samples in the $1-5 \mu \mathrm{L}$ range. $^{22}$

Employing the central element of our previously published design (a polymeric salt bridge) and adapting the electrode configuration featured in similar devices developed by Heineman and co-workers, ${ }^{17-19}$ we have fabricated a simple thinlayer SEC cell that requires no machining for its construction and is capable of analyzing sample volumes on the order of $10^{-8} \mathrm{~L}$. This represents a more than one hundred-fold lesser analysis volume than that of any previously published SEC

Received: December 20, 2012

Accepted: February 19, 2013 
device. In this paper, we describe the design and performance characteristics of this apparatus.

\section{EXPERIMENTAL SECTION}

Reagents. Reagent grade potassium ferricyanide, $\mathrm{K}_{3} \mathrm{Fe}$ $(\mathrm{CN})_{6}$ (13746-66-2), and potassium nitrate, $\mathrm{KNO}_{3}$ (7757-791), were used as received from Fisher Scientific (Pittsburgh, PA, USA). Solutions were typically prepared using distilled/ deionized water and stored in tightly capped polyethylene or polycarbonate bottles. Ferricyanide solutions were deoxygenated by sparging with nitrogen gas prior to use, stored under refrigeration, and discarded if not used within a few days of preparation.

Instrumentation. A BAS Epsilon electrochemical analyzer (Bioanalytical Systems, Inc., Layfayette, IN, USA) was used for electrochemical control and measurement. Spectral measurements were made with a model USB4000-UV-VIS CCD spectrometer and model DH-2000-BAL deuterium/tungstenhalogen light source controlled by SpectraSuite software (Ocean Optics, Inc., Dunedin, FL, USA). A bifurcated fiberoptic transmission dip probe (Ocean Optics model T300-RTUV-vis) with its reflective screw-on tip removed was used to transmit light from source through the sample and deliver light reflected from the working electrode surface to the spectrometer (see lower illustration in Figure 1).

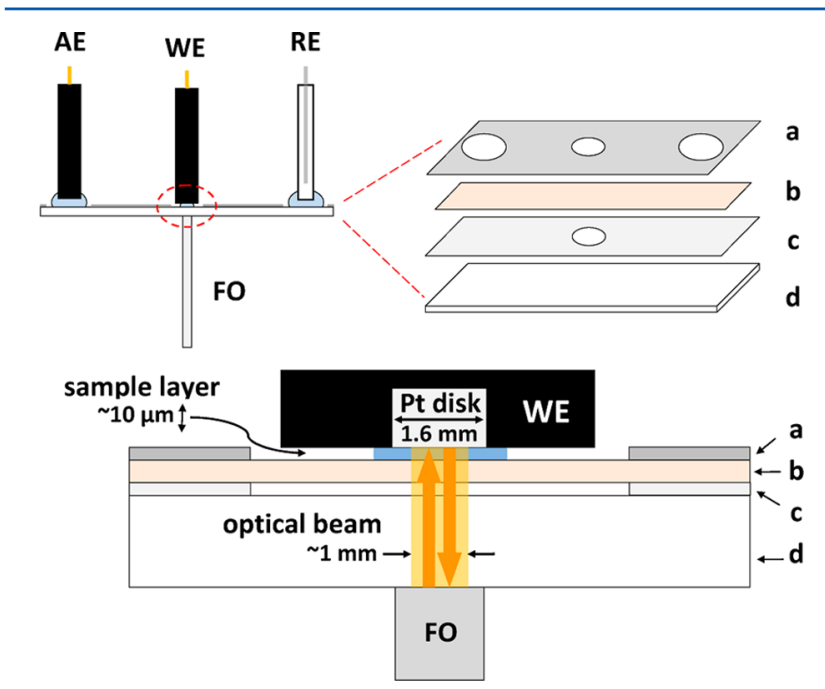

Figure 1. Illustration of the spectroelectrochemical apparatus showing its complete assembly (upper left), an expanded view of the layered substrate platform (upper right), and a zoomed view of the thin-layer sample region (lower, sample-layer thickness greatly exaggerated). AE = glassy carbon disk auxiliary electrode; $\mathrm{WE}=\mathrm{Pt}$ disk working electrode; $\mathrm{RE}=\mathrm{AgCl} / \mathrm{Ag}$ reference electrode; $\mathrm{FO}=$ fiber-optic probe; $a=$ paraffin wax film; $b=$ Nafion film; $c=$ tissue; $d=$ glass microscope slide.

SEC Device Assembly. An illustration of the SEC apparatus is provided in Figure 1. A $2 \mathrm{~cm} \times 5 \mathrm{~cm}$ strip of lint-free cleaning tissue with a $\sim 1 \mathrm{~cm}$ diameter hole cut in its center was soaked with distilled water and placed atop a standard glass microscope slide. A similar size strip of 0.007 in. thick Nafion 117 perfluorinated membrane (31175-20-9, Sigma-Aldrich, St. Louis, MO, USA), previously cut from stock and soaked overnight in $1 \mathrm{M} \mathrm{KNO}_{3}$, was placed atop the tissue strip and secured with a gasket fashioned from paraffin wax film (Parafilm M, Alcan Packaging, Neenah, WI, USA).
This gasket included a center hole matching that of the tissue strip and two larger holes, $\sim 2 \mathrm{~cm}$ diameter, near each end to accommodate electrolyte solution drops for the reference and auxiliary electrodes, and it was cut a bit larger than the microscope slide so that its edges could be folded down and press-sealed to the bottom of the slide. The tissue strip and paraffin film gasket helped maintain the Nafion in a fully hydrated state, a necessary condition for proper operation of the SEC device. When configured for use as detailed below, the cell resistance was approximately $500 \Omega$ as determined by potential-step charging measurements conducted via the electrochemical analyzer's IR compensation utility (though IR compensation was not employed in the voltammetric measurements reported here). Between experiments, the layered substrate platform was stored in a sealed container, either a jar containing a small beaker of water or a zip-lock plastic bag containing a water-soaked tissue or sponge, to prevent dehydration of the Nafion film.

Procedure. The device was configured for use by clamping the platform horizontally on a ring stand and mounting a 1.6 $\mathrm{mm}$ diameter Pt disk working electrode (MF-2013, Bioanalytical Systems, Inc., Layfayette, Indiana, USA) on an $x-y$ translator clamped above the platform. When SEC measurements were planned, the fiber-optic probe was clamped beneath the platform with its terminus flush against the bottom of the microscope slide. With the working electrode raised, a translucent metric ruler was laid across the top of the platform to permit observation of the incident light beam while the fiberoptic assembly was adjusted within its collimator sheath to yield a minimum beam diameter $(\sim 1 \mathrm{~mm})$. The working electrode was then lowered until it made contact with the platform, and its position on the platform surface was adjusted while monitoring the spectral signal until its intensity was maximized. Adequate alignment of the $1 \mathrm{~mm}$ diameter light beam with the $1.6 \mathrm{~mm}$ diameter Pt disk electrode was confirmed by noting that maximum signal could be maintained even as the electrode was repositioned slightly in the plane of the platform. An approximately concentric alignment was achieved by positioning the electrode within this area yielding maximum signal.

Large drops of $1 \mathrm{M} \mathrm{KNO}_{3}$ solution were added to the reference and auxiliary electrode areas on the platform, and these electrodes (RE-5B AgCl/Ag and MF-2012 3-mm diameter glassy carbon disk, respectively, Bioanalytical Systems, Inc.) were mounted securely with their active ends contacting the electrolyte drops. During prolonged experiments, these drops may be easily replenished by adding more electrolyte solution at the electrode perimeters without the need for disassembly. Next, a measured volume of sample solution was added to the sample area of the platform using a fulldisplacement syringe (NanoVolume Model, 1BR-5, SGE Analytical Science, Austin, TX, USA). When configured for SEC measurements, the visible beam spot on the platform served as a convenient marker for accurate placement of the sample drop. Finally, the working electrode was lowered to the desired position and measurements were made. Samples were removed following measurement by a rinse procedure comprised of (a) raising the working electrode; (b) adding a large drop $(\sim 100 \mu \mathrm{L})$ of $1 \mathrm{M} \mathrm{KNO}_{3}$ solution to the sample area; (c) lowering the electrode into the electrolyte drop; (d) raising the electrode; and (e) removing the drop via careful blotting with a lint-free tissue. Subsequent measurements using blank electrolyte solution showed no evidence of sample carryover. 


\section{RESULTS AND DISCUSSION}

The ferri/ferrocyanide couple was chosen to evaluate the performance of our device due to its well-characterized behavior and the anionic charge of both redox species, this latter property serving to prevent analyte loss through permeation into the Nafion film salt bridge per its cation exchange properties. Suitable functioning of the salt bridge was initially confirmed by measurements using relatively large sample volumes $(10-100 \mu \mathrm{L})$ in bulk and thin-layer configurations, the results of which were comparable to those obtained by similar SEC cells previously reported in the literature. We subsequently investigated the use of much smaller volumes $(\leq 1 \mu \mathrm{L})$, making exclusive use of the thin-layer configuration the most practical approach as discussed below.

Aqueous samples in the volume range employed in this work assume a roughly hemispherical shape when placed on the surface of a fully hydrated Nafion film. When the working electrode is lowered on to the sample, it is beneficial to compress the drop to a cylindrical shape in order to increase the electrode/solution contact area and to yield a uniform sample thickness (optical path length) suitable for spectral measurements. Ignoring adhesive and surface tension effects, a simple geometric treatment indicates the maximum sample thickness, $h_{\max }$, will be two-thirds of the original hemispherical drop radius and will exhibit a cube-root dependence on sample volume, $V$ :

$$
h_{\max }=(4 \mathrm{~V} / 9 \pi)^{1 / 3}
$$

Though employing the maximum sample thickness would be desirable from the perspective of spectral sensitivity (optical path length is equal to twice the sample thickness), practical considerations favor the use of minimum sample thicknesses that are achieved by lowering the working electrode fully to make firm contact with the sample platform. The precast Nafion film is prone to buckling during use and does not lay flat against the microscope slide, even when the underlying tissue layer is removed. This is of negligible consequence for relatively thick (large volume) samples but more problematic when working with submicroliter volumes, as resultant deviations in sample geometry and electrode coverage will have a greater relative effect on the measured signals. Pressing the working electrode firmly against the platform effectively flattens the Nafion film and allows for reasonably consistent measurements even with submicroliter volumes. Further, this approach partially compensates for poor placement of sample drops on the device platform, as the expanded radii of compressed samples yield greater coverage of the working electrode surface for drops not positioned concentric with the electrode. Finally, the exposed surface area (perimeter) for cylindrical samples of a given volume is proportional to the square root if its height, so thinner samples will experience a reduced rate of evaporation, often an important consideration in submicroliter sample analysis.

Minimal sample thickness was achieved by lowering the working electrode until it made firm contact with the sample platform. This thickness was estimated photometrically using $20 \mathrm{mM}$ aqueous potassium ferricyanide $\left(\varepsilon_{420}=1020 \mathrm{~L} /\right.$ $\mathrm{mol} \cdot \mathrm{cm}) .^{23}$ A relatively large sample volume, $1 \mu \mathrm{L}$, was employed to ensure consistently complete coverage of the reflective working electrode surface as discussed in the following paragraph. Six replicate absorbance measurements were used to derive via Beer's law an average sample thickness and standard deviation of $8( \pm 1) \mu \mathrm{m}$.

The precision of the manual sample loading procedure employed in this work was evaluated via replicate cyclic voltammetry measurements $(N=6)$ on aliquots of a $20 \mathrm{mM}$ ferricyanide standard solution in the $20-1000 \mathrm{~nL}$ range. Results of these measurements are summarized in Table 1 and show a

Table 1. Precision of Replicate Voltammetric Measurements $(N=6)$ for Varying Volumes of $20 \mathrm{mM} \mathrm{K} \mathrm{K}_{3} \mathrm{Fe}(\mathrm{CN})_{6}$ in $1 \mathrm{M}$ $\mathrm{KNO}_{3}{ }^{a}$

$\begin{array}{ccc}\text { sample volume }(\mathrm{nL}) & \text { avg peak cathodic current }(\mu \mathrm{A}) & \text { \%RSD } \\ 20 & 1.2( \pm 0.7) & 60 \\ 100 & 14.6( \pm 2.4) & 16 \\ 500 & 19.6( \pm 1.1) & 6 \\ 1000 & 22.0( \pm 0.8) & 4\end{array}$

${ }^{a} \mathrm{Scan}$ rate, $20 \mathrm{mV} / \mathrm{s}$.

volume-dependent measurement precision that is likely due to variations in sample placement on the Nafion film, consistent with the assumptions noted above. As the volume decreases, off-center placement of sample drops is more likely to result in partial coverage of the electrode surface upon compression. Also, very small sample drops are more prone to poor delivery and adhesion to the Nafion film since slight twitching of the syringe needle at the time of contact could more easily smear the drop and yield an aberrant geometry. Assuming compression of a hemispherical drop to a cylindrical geometry, a $20 \mathrm{~nL}$ sample compressed to a thickness of $8 \mu \mathrm{m}$ will have a diameter of $1.8 \mathrm{~mm}$. Since the working electrode is a $1.6 \mathrm{~mm}$ diameter Pt disk embedded in a $6.4 \mathrm{~mm}$ diameter insulating sheath, full coverage of the active electrode area will result only if the sample drop is positioned no more than $\sim 0.1 \mathrm{~mm}$ away from the working electrode-concentric point. Larger drops yield larger compressed sample diameters and a corresponding greater tolerance for variations in placement that translates to a greater measurement precision.

The device exhibits excellent thin-layer behavior as evidenced by the cyclic voltammograms shown in Figure 2. The upper traces are voltammograms obtained separately at $1 \mathrm{mV} / \mathrm{s}$ using a blank electrolyte solution and a $3.5 \mathrm{mM}$ ferricyanide standard. The close overlap of background currents in these voltammograms is evident in these overlaid traces and is more explicitly demonstrated in the digitally subtracted voltammogram shown in the lower portion of the figure. The background-subtracted voltammogram displays a high peak symmetry, very small peak separation $(15 \mathrm{mV})$, and negligible diffusion-limited currents (less than $1 \mathrm{nA}$ ). These data were obtained for the smallest sample volume that we were able to reliably deliver per the manual procedure used in this work, $20 \mathrm{~nL}$. In this particular case, the results suggest the sample drop was placed very near the optimal position on the platform as discussed above.

The spectroelectrochemical performance of our device was evaluated through potential-step chronoabsorptometry experiments. Figure 3 shows the change in spectral signal for a $100 \mathrm{~nL}$ drop of $20 \mathrm{mM}$ ferricyanide solution upon repeatedly stepping the working electrode potential from $500 \mathrm{mV}$ (only oxidized form of couple present) to $0 \mathrm{mV}$ (only reduced form present). Absorbance measurements were made at $420 \mathrm{~nm}$, a wavelength at which the oxidized form, ferricyande, absorbs appreciably $\left(\varepsilon_{420}=1020 \mathrm{~L} / \mathrm{mol} \cdot \mathrm{cm}\right)$ but the reduced form, ferricyanide, does not $\left(\varepsilon_{420}=0 \mathrm{~L} / \mathrm{mol} \cdot \mathrm{cm}\right){ }^{23}$ Absorbance values were 


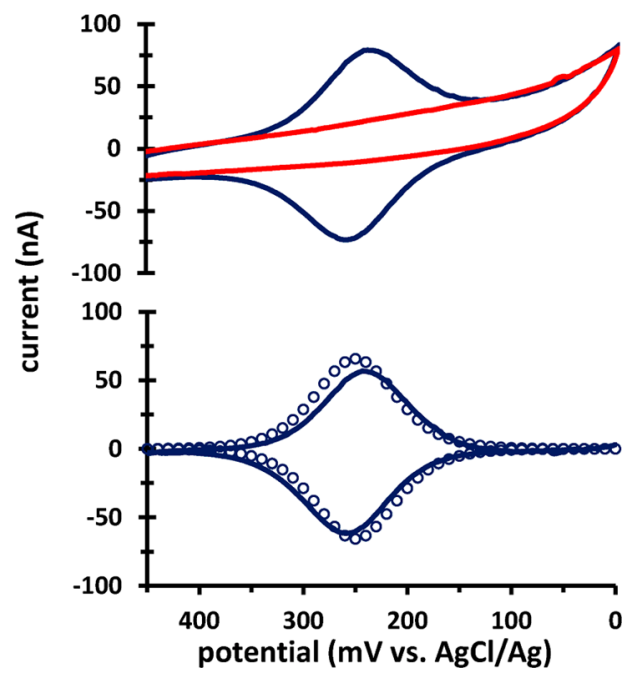

Figure 2. (Upper traces) Cyclic voltammograms for $20 \mathrm{~nL}$ of $1 \mathrm{M}$ $\mathrm{KNO}_{3}$ (red) and $3.5 \mathrm{mM} \mathrm{K}{ }_{3} \mathrm{Fe}(\mathrm{CN})_{6}$ in $1 \mathrm{M} \mathrm{KNO}_{3}$ (blue) measured using the apparatus shown in Figure 1. (Lower traces) Digital subtraction of the two upper traces (solid line) and simulated voltammogram (open circles). Scan rate, $1 \mathrm{mV} / \mathrm{s}$; nominal sample thickness, $\sim 10 \mu \mathrm{m}$.

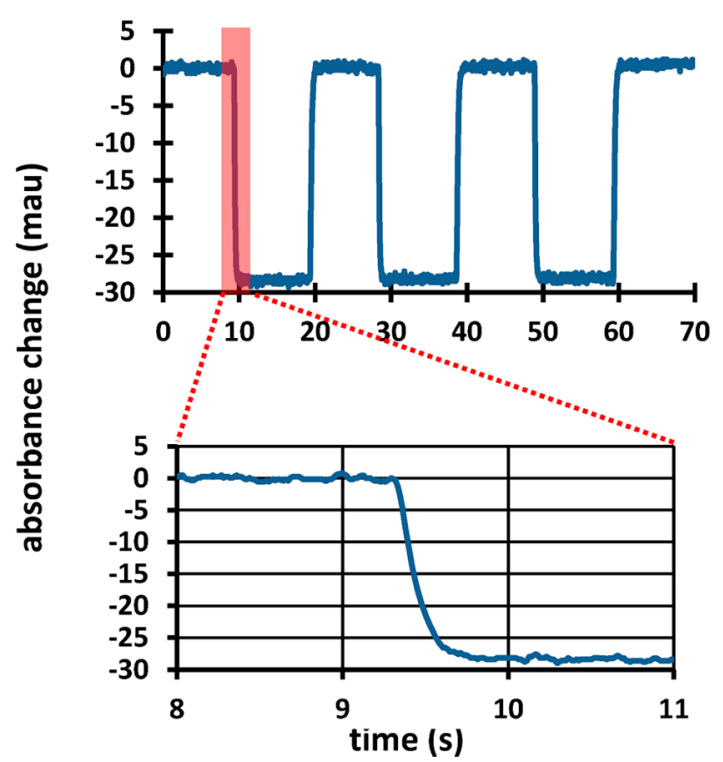

Figure 3. Absorbance change at $420 \mathrm{~nm}$ for $100 \mathrm{~nL}$ of $20 \mathrm{mM}$ $\mathrm{K}_{3} \mathrm{Fe}(\mathrm{CN})_{6}$ in $1 \mathrm{M} \mathrm{KNO}_{3}$ measured upon repetitive potential steps from 500 to $0 \mathrm{mV}$ (13 ms integration time; 21-point smooth; nominal sample thickness, $\sim 10 \mu \mathrm{m}$; reference spectrum acquired at $500 \mathrm{mV})$. A zoomed portion of the first E-step (shaded area) is shown in the lower trace.

computed in potential difference mode using a reference spectrum of the analyte solution equilibrated with the working electrode poised at $500 \mathrm{mV}$. The signal transient depicts a rapid, exhaustive electrolysis of the thin sample layer that is achieved in approximately $0.5 \mathrm{~s}$, in reasonable agreement with the expected time scale based on diffusion within a sample layer roughly $10 \mu$ m thick. $^{9}$

As further demonstration of the cell's SEC performance, exhaustive electrolyses were carried out at several potentials near the analyte's formal potential and the measured spectra used to derive values for the redox couple concentration ratio. These spectra were computed in potential difference mode using a reference spectrum of the analyte solution equilibrated with the working electrode poised at $0 \mathrm{mV}$. Since only one form of the redox couple (ferricyanide) absorbs at the analytical wavelength of $420 \mathrm{~nm}$, the couple's concentration ratio may be computed as

$$
[\text { ferricyanide }] /[\text { ferrocyanide }]=A /\left(A_{500 \mathrm{mV}}-A\right)
$$

where $A$ is the absorbance at $420 \mathrm{~nm}$ measured at any applied potential and $A_{500 \mathrm{mV}}$ is the absorbance measured at $500 \mathrm{mV}$. Assuming Nernstian behavior, a plot of applied potential versus this concentration ratio will be a line whose slope and $y$ intercept give the electron stoichiometry and formal potential for the couple, respectively. Results obtained using a $100 \mathrm{~nL}$ drop of $20 \mathrm{mM}$ ferricyanide solution are shown in Figure 4.

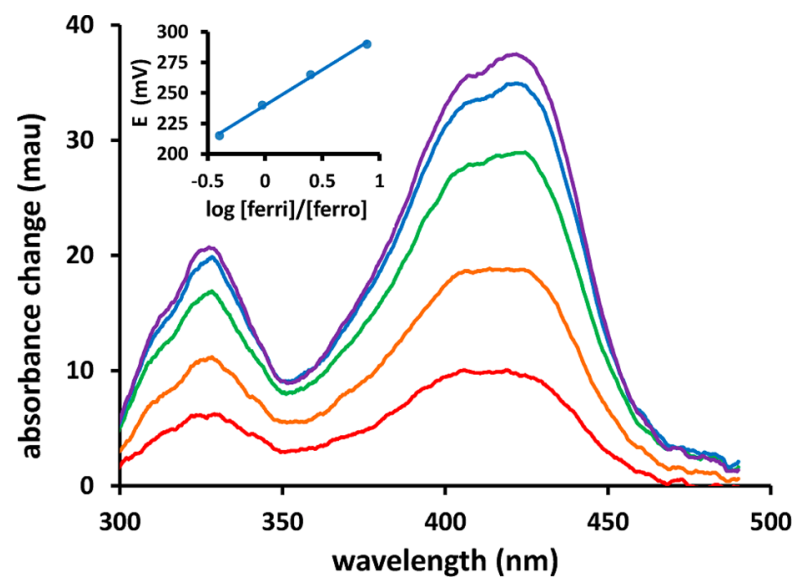

Figure 4. Potential difference spectra for $100 \mathrm{~nL}$ of $20 \mathrm{mM}$ $\mathrm{K}_{3} \mathrm{Fe}(\mathrm{CN})_{6}$ in $1 \mathrm{M} \mathrm{KNO}_{3}$ measured after exhaustive electrolysis at $215,240,265,290$, and $315 \mathrm{mV}$ (10 ms integration time; 400 scans averaged; 21-point smooth; nominal sample thickness, $\sim 10 \mu \mathrm{m}$; reference spectrum acquired at $0 \mathrm{mV}$ ). The inset shows a Nernst plot derived from these spectra.

The Nernst plot generated from these data is linear $\left(R^{2}=\right.$ 0.996 ) and provides a formal potential of $240 \mathrm{mV}$ and an electron stoichiometry of 1.02 , in good agreement with accepted values for this redox couple. ${ }^{24}$

\section{CONCLUSIONS}

In summary, we have described here a novel SEC device that is easily assembled from standard laboratory materials and is capable of reliable, high quality electrochemical and spectroelectrochemical measurements using sample volumes as low as $\sim 100 \mathrm{~nL}$. Volumes as small as $\sim 20 \mathrm{~nL}$ can also be analyzed but with relatively poor repeatability. Proof of concept for the essential component of the device, a polymer electrolyte salt bridge, has clearly been demonstrated, though a few obvious shortcomings in the design may be identified as follows. (1) Poor spectral sensitivity results from the short optical path length associated with transreflectance sampling of a thin sample layer. Improved sensitivity could be realized using a parallel optical sampling scheme similar to the long optical path thin-layer electrode (LOPTLE) designs first described by Kuwana et al. ${ }^{25}$ This would require that the slight buckling of the precast Nafion film be eliminated, e.g., by use of solutioncast films on rigid planar substrates. (2) Use of Nafion as the salt bridge prevents the analysis of cationic analytes (and neutral species with appreciable solubility in the polymer film). 
Analysis of these types of compounds would require the use of an alternative polymer electrolyte in lieu of Nafion. (3) The manual sample loading procedure used is likely the cause of the poor measurement precision exhibited for sample volumes less than $\sim 100 \mathrm{~nL}$. Using micromanipulator-mounted syringes, perhaps with micrometer plunger depression and microscopic observation, could improve sample placement repeatability and enable the use of even smaller sample volumes.

Work in our lab is presently directed toward (a) the development of submicroscale SEC assays for selected anionic species of clinical interest (e.g., ascorbate, urate, bilirubin diglucuronide) and (b) the adaptation of this basic device design to a flow cell configuration that should both address the shortcomings noted above and permit a higher rate of sample throughput.

\section{AUTHOR INFORMATION}

\section{Corresponding Author}

*E-mail: paul.flowers@uncp.edu. Fax: 910-521-6638.

\section{Notes}

The authors declare no competing financial interest.

\section{ACKNOWLEDGMENTS}

Financial support of this work by the UNCP Research Initiative for Scientific Enhancement Program, funded by the National Institutes of General Medical Sciences (grant \# NIGMS5R25GM077634-04), and the Pembroke Undergraduate Research and Creativity Center is gratefully acknowledged. Technical assistance provided in the preliminary stages of this work by students of P.A.F.'s instrumental analysis course in the 2011 and 2012 academic semesters, in particular Trey Archer, is also acknowledged.

\section{REFERENCES}

(1) Kimmel, D. W.; LeBlanc, G.; Meschievitz, M. E.; Cliffel, D. E. Anal. Chem. 2012, 84, 685-707.

(2) Zhang, X., Ju, H., Wang, J., Eds. Electrochemical Sensors, Biosensors and Their Biomedical Applications; Elsevier Science: St. Louis, MO, USA, 2007.

(3) Bowyer, W. J.; Clark, M. E.; Ingram, J. L. Anal. Chem. 1992, 64, 459-462.

(4) Yasukawa, T.; Glidle, A.; Cooper, J. M.; Matsue, T. Anal. Chem. 2002, 74, 5001-5008.

(5) Henry, C. S.; Fritsch, I. J. Electrochem. Soc. 1999, 146, 33673373.

(6) Li, T.; Su, L.; Hu, W.; Dong, H.; Yongfang, L.; Mao, L. Anal. Chem. 2010, 82, 1521-1526.

(7) Ball, J. C.; Scott, D. L.; Lumpp, J. K.; Daunert, S.; Wang, J.; Bachas, L. G. Anal. Chem. 2000, 72, 497-501.

(8) Zoski, C. G. Handbook of Electrochemistry; Elsevier Science: St. Louis, MO, USA, 2007; Chapter 14.

(9) Bard, A. J.; Faulkner, L. R. Electrochemical Methods: Fundamentals and Applications, Second ed.; John Wiley \& Sons: New York, NY, USA, 2001; Chapter 11.

(10) Hawkridge, F. M.; Pemberton, J. E.; Blount, H. N. Anal. Chem. 1977, 49, 1646-1647.

(11) Anderson, J. L. Anal. Chem. 1979, 51, 2312-2315.

(12) Rubinson, K. A.; Mark, H. B., Jr. Anal. Chem. 1982, 54, 12041206

(13) Porter, M. D.; Dong, S.; Gui, Y.-P.; Kuwana, T. Anal. Chem. 1984, 56, 2263-2265.

(14) Luo, H.; Weaver, M. J. Electroanal. Chem. 2001, 501, 141-150.

(15) Ren, B.; Lin, X.; Jiang, Y.; Cao, P.; Xie, Y.; Huang, Q.; Tian, Z. Appl. Spectrosc. 2003, 57, 419-427.
(16) Bonifacio, A.; Millo, D.; Gooijer, C.; Boegschoten, R.; van der Zwan, G. Anal. Chem. 2004, 76, 1529-1531.

(17) Wijayawardhana, C. A.; Halsall, H. B.; Heineman, W. R. Anal. Chim. Acta 1999, 399, 3.

(18) Schroll, C. A.; Chatterejee, S.; Heineman, W. R.; Bryan, S. A. Anal. Chem. 2011, 83, 4214-4219.

(19) Schroll, C. A.; Chatterejee, S.; Heineman, W. R.; Bryan, S. A. Electroanalysis 2012, 24, 1065-1070.

(20) Bistolas, N.; Christenson, A.; Ruzgas, T.; Jung, C.; Scheller, F. W.; Wollenberger, U. Biochem. Biophys. Res. Commun. 2001, 314, 810816.

(21) Caja, J.; Czerwinski, A.; Mark, H. B. Anal. Chem. 1979, 51 (8), $1328-1329$.

(22) Flowers, P. A.; Strickland, J. C. Spectrosc. Lett. 2010, 43 (7/8), $528-533$.

(23) Winograd, N.; Blount, H. N.; Kuwana, T. J. Phys. Chem. 1969, $73,3456-3462$.

(24) Kolfhoff, I. M.; Tomsicek, W. J. J. Phys. Chem. 1935, 39, 945954.

(25) Zak, J.; Porter, M. D.; Kuwana, T. Anal. Chem. 1983, 55, 22192222. 